\title{
ASPECTS DE L'ESCLAVAGE EN ITALIE ENTRE LE IXe ET LE XIIe SIÈCLE.
}

\author{
CHARLES VERLINDEN \\ Professeur ordinaire à l'Université de Gand. Di- \\ recteur de l'Academia Belgica et de l'Institut Histo- \\ rique Belge à Rome.
}

La période examinée dans cet article est comprise entre la chute du royaume lombard et sa conquête par les Carolingiens, d'une part, et le milieu du XIIe siècle, de l'autre. A ce dernier moment les villes italiennes ont déjà derrière elles une évolution assez longue, mais ce n'est qu'alors qu'apparaît avec abondance la documentation spécifiquement urbaine des actes notariés. Ceux-ci forment alors des séries. On possède des registres entiers de notaires dont la pratique reflète l'ensemble de la vie économique et sociale des communes dans lesquelles ils passent leurs actes. Dans ces actes la société urbaine n'apparaît plus comme appartenant à l'époque seigneuriale, mais comme une commune bourgeoise, avec ses diverses stratifications sociales, au bas desquelles l'esclavage prend une place que les actes notariés permettent de connaître de mieux en mieux.

Par contre, pendant l'époque qui suit la disparition du royaume lombard, ce sont les relations de dépendance par rapport au seigneur, individuel ou collectif - dans le cas de communautés religieuses -, qui caractérisent la structure sociale. Ces relations s'étendent, dans le chef des dépendants, à la très grande majorité de la société, mais elles comportent des nuances nombreuses dont les deux principales sont le servage qui monte et l'esclavage, héritier de l'antiquité et de l'époque des royaumes barbares, qui décline.

De même que la période des royaumes barbares est éclairée, pour ce qui regarde la connaissance de la classe servile, avant tout par les sources législatives, ainsi celle qui va, en gros, du IXe au milieu du XIIe siècle l'est-elle par les polytyques et les chartriers 
ecclésiastiques. Nous y voyons quels sont les rapports des monastères, comme seigneurs collectifs, ou des évêques avec leurs dépendants dans les régions italiennes pour lesquelles de pareils documents ont été conservés.

Pour les IXe et Xe siècles les "servi" des grandes propriétés ecclésiastiques italiennes ont été étudiés il y a plus de soixante ans par Gino Luzzatto (1). C'est de son travail que nous devons partir.

Dans le polyptyque de St. Laurent d'Oulx, dans la vallée de Bardonecchia, qui semble dater du Xe siècle (2), il est question de coloni et de provendarii, mais ces deux catégories de dépendants ne présentent aucun trait qui permette de les considerer plutôt comme des esclaves que comme des demi-libres ou serfs. Certes, nous verrons (3) que le terme provendarii implique généralement une dépendance étroite évoquant l'esclavage; mais, à première vue, on serait tenté de penser que dans la région alpine occidentale, voisine des confins de la France actuelle, l'esclavage pur et simple avait dès lors disparu.

Il n'en est pas de même plus à l'est en Lombardie, à Bobbio, dans des fragments d'adbreviationes des années 862 et 883 . Ici apparaissent parmi les res dominicae de la curtis de Cario deux casae de servis (4). Il s'agit évidemment de maisons communes pour les esclaves travaillant les terrae dominicae ou y pratiquant des activités industrielles de type domestique. Les libellarii et les massarii qui figurent à côté des servi sont beaucoup plus nombreux et doivent des redevances en nature, en travail ou en argent du type de celles caractérisant le servage.

Sur les terres de l'évêché de Lucques un relevé de la seconde moitié du IXe siècle mentionne un dépendant spécialisé dans un service proprement domestique dans la curtis de Vecchiano. Il s'agit d'un "Ursus bovarius", mais "habet mansionem et campos et vineas" (5). C'est donc un serf chasé. Faut-il conclure qu'il subsiste encore un peu d'esclavage en plaine, mais qu'il a disparu en montagne et dans les collines au pied de l'Apennin? Sans doute serait-ce prématuré (6).

(1) . - I servi nelle grandi proprietà ecclesiastiche italiane dei secoli IX e X (Pisa, 1910), $190 \mathrm{pp}$. in $8^{\circ}$.

(2). - Ibid., p. 12, n. 1 .

(3). - Cf. ci-dessous p. 55 et n. 34.

(4). - Luzzatto, p. 15.

(5). - Ibid., p. 24.

(6). - D'autant plus qu'encore au début du XIle siècle on achète toujours des esclaves personnels à Lucques. En 1109, le prête Jean paie 8 sous une certaine Insiliola. Cf. Documenti dell'antica costituzione di Firenze, p. 218. 
A Santa Giulia de Brescia le polyptyque est de 905-906. Il a été étudiế par Darmstädter (7) et Seregni (8). On y voit que la réserve seigneuriale était occupée par 741 "praebendarii infra curtem", hommes, femmes et enfants, dont la condition n'est pas absolument claire, mais qui n'en constituent pas moins une troupe servile, plus voisine de celles qu'avait connues l'esclavage antique que de la masse plus considérable des tenanciers demi-libres (9).

Beaucoup plus au sud, à Farfa, en Sabine, nous disposons d'indications précieuses dans les "regestes" et le Chronicon de cette abbaye (10). Cet important établissement ecclésiastique a passé par de longues périodes de violences et de spoliations qui furent parfois le fait de ses propres abbés. Ainsi l'abbé Campone (939-962) non seulement avait donné à ses enfants presque toutes les terres du monastère en Sabine, dans les comtés de Rieti, Amiterno, Forcone, Balva et dans la Marsica, mais il avait, en outre, fait présent à sa femme des curtes de Saxa, S. Benedetto in Silvaplana, San Sisto et Sublongo "cum servis et ancillis quos etiam vendunt et dant (sc. l'abbé et sa femme) filiis et filiabus sicut proprios servos" (11). Aucun doute n'est possible sur la condition de ces "proprii servi" que l'on donne et que l'on vend: ce sont des esclaves. Une liste "De hominibus in Forcone" (12) distingue des "homines cum casa et substantia" qui sont manifestement des serfs chasés, des "homines manuales infra casam in Forcone" et des "mancipia". Il semble bien que ces deux dernières catégories soient constituées d'esclaves. Les "homines infra casam" sont au nombre de onze. Ils ont des fonctions spécialisées: l'un est caballarius, un autre faber, un cocus, deux pistores, cinq hortolani, un aucarius. Deux seulement ont une femme. Dans la même casa résident 55 mancipia, 30 "quae bene laborant", 25 "quae mediocriter laborant". Il s'agit de femmes dont quelques-unes, s'occupant sans doute de l'entretien de l'église, sont qualifiées de "ancillae Dei". D'autres sont mentionnées avec leurs enfants, parmi lesquels il y a 11 "infantes masculi". Les "infantes foeminae" sont mentionnées avec le nom de leur mère et très rarement avec celui du père. Sans doute faut-il conclure

(7). - Das Reichsgut in der Lombardei und Piemont (568-1250) (Strasbourg, 1896) pp. $300 \mathrm{sq}$.

(8). - La popolazione agricola della Lombardia nell'età barbarica (Archivio storico lombardo, t. III, 1895) pp. 39, 88.

(9). - Luzzatto, p. 28 n'est pas suffisamment clair à ce propos.

(10). - Regesto di Farfa (ed. I. Giorgi et U. Balzani, Rome 1879-1882) t. V, no 1280, pp. 254-279 et Chronicon Farfense (ed. U. Balzani, Rome 1903) t. I, pp. 243-301.

(11). - Chronicon I, p. 324 .

(12). - Regesto, t. V, pp. 254-8, Chronicon, t. I, pp. 261-8. 
que le contubernium est encore pour les esclaves domestiques une condition de fait tout à fait courante en Italie centrale au milieu du $\mathrm{Xe}$ siècle.

Toutefois certains serviteurs personnels sont certainement en train d'évoluer vers la demi-liberté. Tel est le cas du cuisinier de Forcone qui a une "substantia" à Pretorio dans le territoire d'Amiterno. "Homo manualis" donc à Forcone, mais serf chasé à Pretorio (13). Il est bien certain que c'est cette dernière condition qui l'emportera chez ses descendants.

Un relevé de dépendants est également conservé pour un monastère beaucoup plus méridional qui passa en 981 sous le contrôle de Farfa. Il s'agit de San Vicenzo al Volturno (14). C'est une adbreviatio de "servi" de la "cella" de Santa Maria di Flaturno, dans les environs de Bénévent, rédigée en 874. On y compte, en tout, 117 servi, mais ils ne sont pas concentrés en una casa comme à Cario sur les terres du monastère de Bobbio, ou comme à Forcone sur celles de Farfa. Ceux de San Vincenzo résident en six endroits différents. De chacun d'eux nous connaissons le nom, accompagné souvent de celui de la mère, rarement de celui du père. Certains sont ferrarius, caprarius, hortolanus. Il y a même un diaconus et deux presbiteri, tandis que l'un des servi est le praepositus de tous les autres. Sont-ce des esclaves? Sont-ce des serfs? L'un et l'autre sans doute, suivant les cas individuels: les clercs et le praepositus plus près du servage, les autres encore pris dans les lacs de l'esclavage. Certains essayent d'y échapper, comme ces hommes dont 1"'origo, remota omni libertate" les condamnait à servir le monastère (15). Mais ces derniers voulaient sans doute se soustraire à l'esclavage puis qu'ils avaient des maisons individuelles et ne résidaient pas tous ensemble dans un édifice appartenant à l'abbaye. Il est vrai que nous ne savons pas où habitaient, dans leurs six habitats différents, les servi de San Vincenzo. Fluidité des conditions, faut-il conclure sans doute, durant cette période qui apparaît de plus en plus comme celle de la transition entre l'esclavage, séquelle de celui de l'antiquité, et l'esclavage différent, conséquence de la traite que nous voyons se développer de plus en plus à partir de la seconde moitié du XIIe siècle. Que, d'autre part, sur le Volturno l'esclavage était encore souvent répresenté on peut le déduire de donations au Monastère de San Vincenzo de terres dont les propriétaires retiennent pour eux-mêmes les servi et ancillae (16), donc dépourvus de terres.

(13). - Luzzatto: op. cit., p. 37.

(14). - Muratori: Rerum italicarum scriptores, T. I, p. II, p. 488.

(15). - M. G. H. Dipl. Karol., n 160.

(16). - Muratori: ibid., p. 380 (803), 387 (833). 
Malgré la fluidité des conditions juridiques, les nécessités de l'économie rurale font que les "homines manuales", les "mancipia infra casam" s'opposent toujours nettement aux "homines cum casa et substantia". Les "rustici", les "coloni", les "massarii", les "manentes" sont toujours nettement distingués de la "familia", des "famuli", des "pueri", "mancipia" "ancillae", "servi", "homines manuales". Les diverses nuances du servage "chasé" s'opposent clairement à l'esclavage personnel (17). A Arezzo dans une donation de 840 on énumère d'abord les terres des colons avec les familles qui y résident, puis il s'agit "de vero personis hominum dedimus quattuor personas manuales, pistorem, cocuum, lavandarium et befulcum" (18). Il est curieux de noter que ces "personae" sont beaucoup plus loin de la personnalité juridique que les colons, dont la terre garantit l'indépendance relative. De même, beaucoup plus au sud, le Chronicon $\mathrm{Ca}$ sauriense abruzzais (19) fait état d'une vente de 856 où s'opposent sur deux curtes les "cartulati vel coloni cum colonicis suis" et les "servos et ancillas vel omnia et in omnibus quicquid nos paratum habemus". Ces derniers, on le voit, sont confondus, comme les esclaves antiques, avec les choses. Il en est de même pour les "servi vel ancillae qui nobis in casa servierunt" à Farfa en 802 (20) et, encore plus clairement, dans le Nord, à Biella, en 826 , où il s'agit d'un "mansum dominicatum cum casa dominicata et aliis aedificiis et mancipiis desuper commanentibus" (21). Encore en 1034, près de Forlì en Romagne, dans un échange de dépendants, il est fait exception de "illis qui assidue de domnicata stipendia vivunt" (22). Ces derniers sont des esclaves personnels qui sont entretenus par le maître.

Pour se procurer des esclaves personnels les seigneuries ecclésiastiques avaient parfois recours à la traite et nous aurons l'occasion de revenir sur le rôle international qu'y jouaient dès lors les Vénitiens et les Amalfitains. Il en était d'ailleurs de même dans d'autres régions. Ainsi, en 1007, le couvent de San Benedetto de Conversano dans les Pouilles obtient du catapan de Bari le privilège de ne payer aucun "praetium commercii de quantiscumque mancipiis comparaverunt in eadem civitate Baro pro servitio eiusden monasterii" (23).

Beaucoup de ces esclaves ont des tâches bien déterminées. A Sienne il s'agit en 867 d'un "pistrinarius", d'un "lavandarius" et de

(17). - Luzzatto: op. cit., p. 79.

(18). - Pasqui: Codice diplomatico aretino, $\mathrm{n}^{\circ} 30$.

(19). - Muratori, t. II, p. II, col. 926.

(20). - Regesto di Farfa, t. II, p. 173.

(21). - Muratori: Antiquitates Italiae medii aevi, t. V, col. 553.

(22). - Ibid., t. I, col. 589.

(23). - Morea: Chartularium Cupersanense, $n^{\circ} 63$. 
trois "tappetarii" (24). En 929 le roi Hugues confirme au monastère de San Pietro in Ciel d'Oro de Pavie une donation faite par son prédecesseur lombard Liutprand des "carpentarii quos praedictus locus dinoscitur possedisse in valle Autelam vel in vico Besogolo cum filiis filiabusque vel eorum agnatione ut tempore opportuno indefesse operando deserviant ii vel posteri eorum in supradicto coenobio" (25). Dans ce dernier cas, l'évolution vers le servage paraît cependant certaine. Il s'agit, en effet, de dépendance héréditaire et celle-ci est une caractéristique essentielle du servage. La situation observée dans les terres de San Pietro in Ciel d'Oro est analogue à celle que l'on peut suivre avec tant de précision dans certaines généalogies serviles espagnoles un siècle plus tard (26). A Pavie il n'est pas impossible que l'allusion au "tempore opportuno" auquel doivent se faire les prestations des charpentiers vise la transformation d'une tâche permanente (indefesse) en une corvée à temps, fixée par la coutume. Ailleurs la situation est plus claire et plus nette. Ainsi pour le "pistor" et le "lavandarius" cités à Ravenne et à Arezzo, en 973 l'un, en 840 l'autre (27); ou encore pour un "textor" et un "pigmentarius" que l'on trouve à Tarente en 1114 (28). Ces artisans non-libres auraient été des "idonei" à l'époque des royaumes barbares et auraient valu plus qu'un simple esclave rural. A présent, ils sont restés attachés à leur métier servile, tandis que l'attribution d'une terre a transformé la condition des "servi casati".

Les femmes sont encore souvent groupées dans le "genitium" ou "pisele". A l'abbaye de Nonantola en Lombardie en 895 les esclaves occupées au tissage devaient être nombreuses, car on peut se permettre d'en envoyer chanque année douze au monastère dépendant de San Michele Arcangelo à Florence, pour y faire des chemises de laine et de lin (29). A Vérone l'évêque Rataldo donne aux chanoines le dixième des vêtements "quae de pisile veniunt vel de gineceo" (30).

Les femmes des gynécées sont, elles aussi, des ouvrières spécialisées, mais à côté d'elles et des artisans masculins non-libres que nous avons déjà mentionnés, il y a de multiples travailleurs des champs

(24). - Luzzatto, p. 82.

(25). - Monumenta Historiae Patriae, t. XIII, $\mathrm{n}^{\circ} 529$.

(26). - Cf. C. Verlinden: L'esclavage dans l'Europe Médiévale t. I, pp. 122 sq. n० 35

(27). - Fantuzzi: Monumenti ravennati, t. I, $\mathrm{n}^{\mathrm{9}}$ 48; Pasqui: op. cit.,

(28) . - Gattola: Ad historiam abbatiae Cassinensis accessiones (Venise, 1744) p. 231.

(29). - Tiraboschi: Storia dell'Abbazia di Nonantola, App. n 53.

(30). - Ughelli-Coleti: Italia sacra, t. V, col. 605. 
formant encore des groupes que l'on déplace suivant les nécessités du travail. Ils étaient souvent réunis en "ministeria". A Bobbio, d'après l'ordonnance de l'abbé Wala de 834-836, il y a le ministerium du praepositus qui règle le travail sur les champs et pâtures, celui du cellerarius familiae qui fournit le vin des travailleurs agricoles, ceux des camararii qui surveillent les "sutores calciamentorum ac vestimentorum", les "compositores pellium", les "calderarii", les "fabri scutarii"; celui, enfin, du praepositus junior qui commande les "operarios praeter eos qui in diversis officinis deputati sunt" (31). Ce sont évidemment les servi du praepositus et du praepositus junior qui devaient être le plus souvent déplacés suivant les nécessités du travail.

De pareils esclaves sont entièrement à charge du maître pour leur entretien, et nous avons déjà mentionné le document romagnol de 1034 où il est question de "illis qui assidue de domnicata stipendia vivunt" (32). Que ce soit là une caractéristique de l'esclavage, on le voit fort bien dans un acte de 1031 par lequel une Amalfitaine du nom de Gemma vend son "infantulum" à un nommé Giovanni Tecapanta qui pourra le garder toute sa vie et lui ordonner tout ce qu'il voudra "die et nocte, longe et prope, per mare et per terram", mais devra le "nutricare et vestire et calzare iusta ratione et secundum suam possibilitatem" (33). Nul doute que l'infantulus ne soit un esclave, et pas davantage que, tout comme les servi romagnols de trois ans plus tard, il ne vive "assidue" des "stipendia" de son maître. Souvent ces "stipendia" s'appellent "provenda" ou "praebenda" et ceux qui les reçoivent "praebendarii". Il devient donc tout à fait évident à présent que les praebendarii de Santa Giulia de Brescia, au début du Xe siècle, dont il a été question plus haut sont bien des esclaves travaillant en groupes (34). Parmi ces praebendarii il y a une majorité de femmes et d enfants. Cependant la famille n'est pas légalement reconnue parmi eux (35).

La famille naturelle n'est pas davantage respectée par les maîtres. A Ravenne, en 973, le duc Pierre donne au monastère de San Apollinare Nuovo son servus Ursellus, bouvier, avec ses enfants "excepto Johannes pistore, filio ipsius Urselli befulci, quem ad meis reservo manibus" (36). Ou, encore, à Salerne, en 966, l'abbaye de la Cava

(31). - Hartmann: Die Wirtschaft des Klosters Bobbio im 9. Jahrhundert (Analekten zur Wirschaftsgeschichte, Gotha, 1904) App. IV, p. 130.

(32). - Ci-dessus p. 53.

(33). - Codex diplomaticus Cavensis, t. V, n 833.

(34). - Cf. ci-dessus p. 3; de même, sans doute, pour les "provendarii" de St. Laurent d'Oulx. Ct. ci-dessus p. 50.

(35). - Luzzatto, op. cit., p. 99.

(36). - Fantuzzi: op. cit., t. I, no 48. 
aliène une "Regauda mancipia" dont il est dit qu'elle a "unum filium masculinum quod non dederunt sed reserbaverunt ad postestatem ipsius ecclesiae faciendum de illius persona omnia quod voluerint" (37). Pour les maîtres il s'agit de biens meubles. Ainsi à Bari en 1103 il est question de "quocumque mobile excepto duos servos meos" (38). On échange les "servi" personnels (39); on les troque contre une terre (40); on les donne en gage (41), on les constitue en dot (42).

Comme les esclaves antiques les servi personnels pouvaient posséder un pécule souvent appelé "paratum et conquisitum". Ainsi en 1063, à Salerne, un certain Romuald affranchit devant le juge son ancilla et ses trois enfants et leur concède "omne illorum iustum paratum et conquisitum ad faciendum ex eo quod voluerint" (43). Ce pécule consistait en biens meubles ou en argent, jamais en terre. Il provenait du travail artisanal ou agricole du servus ou de l'ancilla, fait en dehors du service ordinaire dû au maître, mais avec l'autorisation de celui-ci. Il pouvait aussi résulter de dons du maître, ce qui était plus rare, mais se présentait parfois pour des femmes et pour des motifs faciles à deviner.

Tous ces traits sont la continuation de ceux qui caractérisaient l'esclavage dans les royaumes barbares d'Italie et antérieurement sous l'Empire. Mais ils n'étaient plus désormais caractéristiques que d'une minorité. A Santa Giulia de Brescia, il y avait 741 praebendarii ou esclaves sur un total de 4.700 dépendants. La proportion était donc de 1 à 5 en faveur du servage. Sur la terre de Forcone que l'abbaye de Farfa possédait dans les Abruzzes, il y avait 93 personnes "infra casam" sur un total de 1.500 dépendants. Ici la proportion était de 1 à 14 (44). L'eclavage reculait donc tandis que les serfs augmentaient en nombre par l'acquisition de terres (45). Celles-ci leur étaient

(37). - Codex Cavensis, t. II, n० 244.

(38). - Codice diplomatico Barese, $t$. V, no 36.

(39). - En 926, par exemple. Cf. Codex Cavensis, t. II, n' 244.

(40). - A Vérone en 944. Cf. Muratori: Antiq. Ital. Medii Aevi, t. $\mathrm{V}, \mathrm{col} .605$.

(41). - A Bari en 1130 (Codice diplomatico Barese, t. V, n ${ }^{9} 79$ ) ou encore en 1141 où il est question d'un Mauro de Ravello "qui malo ordine pignoravit unum molinum meum et unam ancillam meam nomine Negizza, quae de propria causa mea comparata fuerunt" (ibid., n? 94).

(42). - Exemples à Bari en 1002, 1057, 1065, 1130, 1137 (Cod. dipl.

Barese, t. IV, $n^{\circ} 18, n^{\circ} 36 . n^{\circ} 42 ; t$. V, $n^{\circ} 78, n^{\circ} 87$

(43). - Codex Cavensis, t. VIII, n' 1359.

(44) . - Luzzatto, p. $111 \mathrm{n}$. 1 commet quelques erreurs à ce propos.

(45). - Ou parfois par simple émancipation. Voici un acte du 3 janvier 1091, par lequel Arnaldus, prêtre de Novare, affranchit son esclave Albert par testament (Historiae Patriae Monumenta, Chartarum t. II, col. 693): "et hoc 
souvent données par les maîtres, au service desquels ils demeuraient du reste en changeant de condition. En réalité, bien des maîtres considéraient que c'était là pour eux un avantage, car ils n'avaient plus désormais à entretenir le serf qui leur devait toujours des corvées, c'est-à-dire du travail réglé par la coutume, ou bien des prestations en nature ou en argent.

Telle était donc, dans ses grandes lignes, l'évolution qui, à l'époque que nous étudions, se marquait dans une partie considérable de l'Italie: l'esclavage, hérité de l'antiquité, se transformait en servage.

Mais, en même temps, la traite reprenait une importance peu à peu croissante qui se reflète tout d'abord dans les noms et la nationalité des servi. Des noms en eux-mêmes on ne peut évidemment conclure grand'chose, car ils se modifiaient suivant les prédominances politiques successives. Ainsi on rencontre à Forcone dans les Abruzzes une grosse majorité de noms germaniques, dont beaucoup sont de forme très probablement ostrogothique (46). Il serait, toutefois, erroné de croire que ceux qui les portaient étaient nécessairement des descendants d'Ostrogoths, d'autant plus que le document qui les mentionne date du $\mathrm{Xe}$ siècle!

Plus intéressants sont des documents de l'Italie méridionale montrant que des servi venant de fort loin y étaient présents. Sans doute au moins une partie d'entre eux y étaient-ils arrivés par la traite. Ainsi l'abbaye du Montcassin possède, entre autres, à Tropea en Calabre, un "Costa longobardus" en 1090 (47). Plus tard, à Tarante, il s'agit de la donation de "Joannes saracenus, Theodorus grecus, Demetrius grecus, Dragius bulgarus, Costa grecus, Theodorus armenus, Simeon bulgarus et Maria bulgaria" (48). C'est assez pour qu'il

volo meo addere testamento ut Albertus mei iuris servus sit liber de sua persona ab omni servicio, et tamen, si voluerit habitare cum suprascripto Glirardo vel cum presbitero qui fuerit electus in suprascripta ecclesia, semper habeat victum et vestitum ex beneficiis ecclesie et faciat eis servicium quod scit et possit. Et si cum eis habitare et servire sibi aptum non fuerit, ex beneficiis suprascripte ecclesie per unumquemque annum habeat solidos decem mediolanensium denariorum. Et si postquam se a suprascriptis separaverit et ad eius vel eorum successores redire voluerit, et, ut diximus, servire diebus vite sue, victum et vestimentum ex beneficiis suprascripte ecclesie non ¿isint sibi". Albertus n'est évidemment plus esclave personnel après son ímancipation, mais il est, entendu qư'il restera attaché à l'église qui continuera à assurer son entretien.

(46). - Luzzatto, p. 186.

(47). - Gattola: op. cit., p. 34.

(48). - Ibid., p. 231. Les Sarrasins étaient parfois faits prisonniers et ensuite asservis sur les côtes italiennes elles-mêmes. Voici un exemple de 849 extrait du Liber Pontificalis (Vita Leonis IV, CLIV) (éd. Duchesne, t. II, p. 
vaille la peine de consacrer quelque attention particulière aux régions de l'Italie méridionale qui allaient constituer beaucoup plus tard la partie continentale de l'ancien royaume de Naples.

Pour cette région nos données les plus anciennes remontent au Xe siècle (49). Le tome Ier des Regii Neapolitani archivi Monumenta contient un acte de 928 par lequel un dénommé Stephanus Mannociolus promet à son père Maurus de ne pas réduire "in servitutem" après sa mort, certains "famulos" que celui-ci avait affranchis (50). Le fait que "servitus" vise bien ici l'esclavage est rendu évident par un passage du même acte où il est dit que les affranchis de Maurus avaient été achetés aux Sarrasins (51). Il est donc certain qu'ils ont été asservis par ceux-ci en tant qu'esclaves. Il semble même que Maurus a dû les posséder pendant quelque temps dans les mêmes conditions, car si son fils n'avait pas su qu'ils avaient été esclaves de son père, celui-ci n'aurait pas dû lui faire promettre qu'il respecterait leur liberté: D'autre part, il paraît bien aussi qu'une fois affranchis ces anciens esclaves soient restés des famuli, c'est-à-dire des dépendants dont la subordination à leur maître correspondait à l'un ou l'autre dégré du servage. Dans le cas qui nous occupe, il est clair que ce lien

119) concernant des prisonniers faits par les Romains et leurs alliés d'Italie méridionale lors d'une bataille navale au large d'Ostie: "Ex quibus (= les Sarrasins) per quasdam insulas nostras famis laborantes penuria plurimi a nostris hominibus interfecti sunt, ceteros autem vivos ... conprehendentes, Romam duxerunt. Quorum Romani proceres, ne multiplicatus numerus videretur, multos prope Portum nostrum Romanum in ligno suspendi iusserunt; aliquantos etenim nos ferro constricti vivere iussimus, pro hac solum modo causa ut et spem nostram, quam in Deum habemus, et illius ineffabilem pietatem, necnon et propriam tyrannidem luce clarius scire valuissent. Et post haec, ne otiose aut sine angustia apud nos viverent, aliquando ad murum quae circa ecclesia beatissimi Petri apostoli habebamus inceptum, aliquando per diversa artificum opera, quicquid necessarium videbatur, per eos omnia iubebamus deferri. Pro his ideo beneficiis, ut iam enarratum est, eos fecimus reservare".

(49). - Lokys: Die Kämpfe der Araber mit den Karolingern bis zum Tode Ludwigs II (Heidelberg, 1906) p. 24 fait cependant allusion au commerce d'esclaves pratiqué à Naples au IXe siècle.

(50). - T. I (Naples, 1845), p. 44.

(51). - Dès la fin du VIIIe siècle les razzias musulmanes sur la côte napolitaine sont fréquentes. Cf. p. ex. les passages de la Chronique des ducs de Bénévent, Salerne, Capoue et Naples, reproduits dans les Monumenta ad Neapolitani ducatus historiam pertinentia, t. I, (1881), p. 65 où l'on voit en 788 les Sarrasins "Neapolim eiusque vicinos agros depopulavisse". P. 69 (801) figure la mention suivante "Mauri... in Pontias et Aenariam insulas mense augusto egressi, earum incolas occiderunt, agros devastaverunt, bona diripuerunt et cum plurimis captivis secum abstulerunt, sine ulla Neapolitarum, quibus altera illarum insula (sc. Aenaria $=$ Ischia) pertinebat, oppugnatione". 
dérivait de l'obsequium dû par l'affranchi (52). Nous nous trouvons donc très vraisemblablement devant un cas de passage de l'esclavage au servage par voie d'affranchissement. De plus, nous l'avons vu, la traite a joué ici son rôle.

Malheureusement le sens du terme famulus n'est pas toujours aussi clair dans les textes des hautes époques. Ainsi, dans un testament de 968, une religieuse napolitaine. retirée au couvent après son veuvage, fait différents legs à des individus qualifiés de famulus on famula. Sont-ce des esclaves? Il ne semble pas, car, sinon, ils ne pourraient pas acquérir de biens. Sont-ce des affranchis? Sans doute, car un passage du testament dit que la testatrice "relinquit liberos omnes famulos et famulas suas" (53). Mais de quelle sorte de dépendance ont-ils été libérés? De l'esclavage? D'une des nuances du servage? Nous ne savons.

Si le sens de famulus n'est pas toujours évident dans les documents napolitains du Xe siècle, il en est souvent de même pour servus et. ancilla. En 973, un certain "Stephanus cognomento Basapicculus" a affranchi par testament son servus Pierre et son ancilla Marie, en leur accordant l'usufruit d'une maison dont le monastère des SS. Serge et Bacchus retiendra le dominium (54). Ces affranchis étaient-ils auparavant des non-libres, c'est-à-dire des esclaves, ou des demi-libres, c'est-à-dire des serfs? Le texte que nous venons d'analyser ne permet pas d'en décider; mais le hasard qui préside à la préservation des documents nous a ici favorisés, car un autre acte de la même année (4 février) nous en apprend davantage. C'est un "dispositum factum a Stephano, cui supranomen Basapicculum, de omni sua substantia" (55). Il s'agit donc du testament même auquel il était fait allusion dans le texte précédent. La première disposition concerne notre ménage d'affranchis. Voici la formule d'affranchissement: "Primum omnium disponit quod Petrus et Maria, servus et ancilla, post eius obitum permaneant liberi pro anima sua cum omnibus ipsorum peculiares et bestimentis seu stromaciolis: sed qui ex eis, si ancillam aut servum uxorem vel virum tulerit, aut se servo vel ancille tradiderit vel succuba introierit, portionem eius apprehendat abbas monasterii SS. Severini et Sosii". L'affranchissement porte donc également sur le pécule, les

(52). - Des situations semblables peuvent s'observer en Espagne. Cf. C. Verlinden: La condition des populations rurales dans l'Espagne médiévale dans Recueil de la Société Jean Bodin (2e éd. Bruxelles, 1959), p. 180.

(53) . - B. Capasso: Monumenta ad Neapolitani ducatus historiam pertinentia, t. II, Ie part. (Naples, 1885), p. 108, n' 164.

(54). - Regii Neapolitani archivi Monumenta t. I, part. II (Naples, 1847) p. 217, no CL. L'affranchissement est indiqué par la formule "testamento. . in libertatem vindicat".

(55). - B. Capasso: op. cit., p. 124, no 196. 
vêtements et la literie, ce qui semble convenir assez bien pour des esclaves proprement dits. D'autre part, il apparaît que le maître désire très vivement préserver le nouveau statut qu'il a accordé à Pierre et Marie, car si ceux-ci s'unissent à un servus ou une ancilla, ils perdent les humbles biens dont ils sont à présent propriétaires, en faveur de l'abbaye qui semble bien exercer à leur égard les droits du patronat. La limitation de la liberté du choix dans le mariage et cette rétention éventuelle de la succession ou d'une partie de celle-ci par le seigneur - ici l'abbaye - sont caractéristiques du servage. Cette constatation est de nature à renforcer la vraisemblance de l'interprétation proposée ci-dessus: il doit s'agir d'esclaves entrés par voie d'affranchissement dans une dépendance assimilable à l'une des nuances du servage. Il ne semble pas téméraire de voir une indication allant dans le même sens, dans le fait que le testament ne mentionne pas seulement le mariage, mais aussi des formes d'union fort analogues au contubernium servile.

Sans doute y a-t-il quelque raison d'admettre, malgré le caractère flottant du vocabulaire latin relatif à la structure de la classe servile à cette époque, que servus doit avoir eu assez souvent son sens classique. Voici, par exemple, un texte de 954 se rapportant à Gaète (56) . Une "serva" y est donnée "cum duobus infantulis suis", ce qui évoque tout naturellement l'esclavage. De même un document de 996 figurant dans le Codex diplomaticus Cavensis (57) contient un affranchissement à temps de deux famule et d'un servus (58) qui ressemble fort à des stipulations analogues concernant, dans l'antiquité ou à la fin du moyen âge, des individus dont la condition est indubitablement l'esclavage. Mais à nouveau le caractère vague de la terminologie sociale, dans cet âge de transition que constitue le Xe siècle, est souligné par le fait que nous trouvons deux termes différents pour désigner des gens de condition identique. L'une des famule s'appelle "Polcara", ce qui laisse soupçonner qu'il s'agit d'une esclace d'origine bulgare.

Les indications sur l'origine ethnique des esclaves sont fort rares au Xe siècle. Le Codex diplomaticus Cavensis fournit néanmoins un renseignement curieux dans un acte de 966 où l'on voit le prêtre Jean donner à l'abbé de S. Massimo deux mancipii, Marie et Pierre, "qui

(56). - Codex diplomaticus Gaetanus (Montecassino, 1887-1891) $\mathrm{n}^{\circ} 52$.

(57). - T. III (Milan, 1876) $\mathrm{n}^{\circ} 491, \mathrm{p} .46$.

(58). - "Tantum Pretiosola et Polcara, famule mee, et Petrus, servum meum, deserviant ipsi Rogate filie mee, dum viba fuerit; post obitum ipsius filie mee, libere et liberos absolutos vadant cum sua conquisita et cum quantos filios et filias infra hanc audientia fecerint". 
fuerunt ex genere Francorum" (59). Il s'agit sûrement d'habitants du nord de l'Europe faits prisonniers et asservis au cours d'un conflit armé et vendus ensuite par des traitants. Ces esclaves sont échangés contre une autre "mancipia" dont le fils reste "ad potestate ipsius ecclesie faciendum de illius persona omnia quod voluerit" (60). Mais, répétons-le, s'il semble certain que nous ayons affaire ici à des esclaves, il est d'autres où les servi cités dans les documents sont manifestement des serfs, c'est-à-dire des demi-libres. Ainsi un document de 999 concerne des servi qui s'étaient refusés à faire le servitium pour l'évêque de Gaète, protestant "ut nullo modo essemus vestri servi, sed veri liberi" (61). Le missus de l'empereur à qui l'évêque avait eu recours donne aux servi trois jours pour décider s'ils voulaient "pugnare ad spatham", c'est-à-dire soutenir un combat judiciaire comme des hommes libres, ou bien se reconnaitre serfs. "Pro magno pavore" ils refusent le combat et n'osent prouver par les armes que leur père "servitium de episcopio non fecisset sicut alii massarini". Ils se reconnaisent donc serfs ou massarini. Il s'agit évidemment ici de serfs chasés, pourvus de terres, c'est-à-dire jouissant d'une certaine aisance économique. Ils se rendent compte d'ailleurs que c'est là que réside leur force réelle et que c'est en se rachetant qu'ils feront reconnaître en droit la liberté dont ils jouissaient déjà en fait par suite de leur situation économique privilégiée. Aussi se rachètent-ils par la suite en payant une livre "de auro purissimo".

A partir du Xe siècle, nous l'avons vu, le terme famulus apparaît à côté de servus, ancilla, mancipium. D'autres termes encore désignent en Italie méridionale les diverses nuances de la demi-liberté; tels sont clientulus, infantulus, defisus, commenditus, affidatus, offertus, censitus, hospitus, excusatus. A. Lizier qui les relève (62) y voit une preuve de l'amélioration de la condition du servus, de son "elevazione morale", de l'"indebolimento dei suoi vincoli servili". Ne serait-ce pas aussi que l'on éprouve de plus en plus le besoin de distinguer le "servus", devenu serf, de l'esclave proprement dit d'origine étrangère?

Nous avons rencontré déjà une "Polcara", sans doute bulgare, de même que des "mancipii ex genere Francorum". Voici en 1057, provenant du Codice diplomatico Barese (63), une promesse de ma-

(59). - Cité par A. Lizier: L'economia rurale dell'età prenormanna nell'Italia meridionale (Súdi su documenti editi dei secoli IX-XI, Palerme, 1907), p. 60 .

(60). - Lizier: loc. cit.

(61). - Op. cit., p. 63, n. 5 .

(62). - Op. cit., pp. 65 sq., 73.

(63). - T. IV. Le Pergamene di S. Nicola di Bari. Periodo greco (939-1071) (éd. F. Nitti di Vito, Bari, 1900) no 36, p. 75. 
riage mentionnant "unam ancillam Zitam, bonam, ex genere Sclab()rum, cum membris sanis, apta ad omnem imperationem faciendum". Aucune hésitation n'est possible, il s'agit d'une esclave slave, ce qui ne doit pas surprendre dans les Pouilles, si proches des Balkans. D'autre part, les chrétiens d'Italie méridionale razzient à présent des musulmans. Victimes autrefois des raids sarrasins, ils ont passé à l'offensive. A preuve cette "ancilla, Setanna nomine, cum suo filio Nicolula ex genere Sarracenorum" qui fait partie d'une dot en 1065 (64). Le petit esclave semble même baptisé, vu son nom, mais cela n'a pas suffi à l'affranchir.

Sous le régime normand les réductions en esclavage se multiplient. Assurément nous ne savons pas s'il s'agit réellement de pareil asservissement dans une donation de 1102 que les Regii Neapolitani archivi Monumenta edita ac illustrata (65) résument de la façon suivante: "Rogerius, Calabriae comes, Brunoni monacho donat, ut pote servos perpetuos et villanos, centum duodecim Syllacenses ac Suberatenses, qui in Capuae obsidione cum Sergio in ipsum Rogerium conjunctionem iniverant". La présence du terme villanos dans ces regestes ne suffit pas à en exclure l'hypothèse, mais aucune certitude n'est permise. Sans doute, se trouve-t-on sur un terrain plus ferme quand on voit en 1119 Robert, prince de Capoue, donner au monastère de S. Biagio deux homines avec leurs descendants et leurs biens "ad possessionem et potestatem et dominationem ipsius monasterii Sancti Blasii et eius custodibus et rectricibus, ad habendum et possidendum et faciendum ex eis quicquid vobis placuerit". Cette dernière clause, en tout cas, paraît viser des esclaves (66). Aucun doute n'est permis à propos d'une vente de 1121 (67). On y voit un nommé Constantinus Calabriensis, de Bari (intus civitate Bari), vendant à un concitoyen du nom de Petrus de Laudula "unam ancillam meam, nomine Dragam, cum una infantula, filia sua, nomine Ducatella, que ancilla fuit ex genere Sclavorum". Le prix est de "quinquaginta et sex miliarenos de ramesinis bonis". Le vendeur garantit la propriété de l'acheteur "in ea videlicet ratione ut a presenti die in antea ipsa predicta ancilla et iamdicta filia eius sint transactive in potestate et dominatione tua et

(64). - Ibid., n n $^{\text {42, p. } 83 .}$

(65). - T. V, (Naples, 1857), no DX.

(66) . - A. Gallo: Codice diplomatico Normanno di Aversa (Naples, 1927) (Società napoletana di Storia Patria. Documenti per la storia dell'Italia meridionale), p. 355 .

(67). - Codice diplomatico Barese t. V. Le pergamene di S. Nicola di Bari (Periodo normanno, 1075-1194) (éd. F. Nitti di Vito, Bari, 1902) n? 66, p. 114 . 
tuorum heredum, habendi, dominandi, possidendi et omnia ex inde faciendi ut vestra erit voluntas, sine requisitione et contrarietate mea et meorum heredum omniumque hominum". Il s'agit déjà d'un acte de vente devant notaire et témoins (68) comme on en rencontrera de plus en plus pendant les derniers siècles du moyen âge (69).

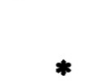

(68). - "Et hanc cartulam venditionis scripsit Conto protonotarius qui interfuit"; suivent les noms des témoins.

(69). - On rencontre des actes notariés vénitiens concernant la traite antérieurs à ou contemporains du texte de 1121 de Bari cité ci-dessus. Il existe toutefois des actes de vente notariés d'esclaves beaucoun plus anciens. Voici un acte passé à Asti en 926 relatif à la vente d'un "servus" sous-diacre à l'évêque d'Asti. Cette vente est vraisemblablement le moyen que le propriétaire, prêtre lui-même, a trouvé pour acheminer le sous-diacre vers la liberté. Voici ce texte rédigé en un latin assez barbare: "Constat me Daniel, presbiter de loco Carenciano, vivente lege romana, acepisem sicuti et in presencia testium acepi, ad vos domnus Audax. episconus Sancte Astensis Ecclesie, per misso vestro, Benedictus preshiter de loco Valeriano, argentum et mercis valente solidos tresgenti, fenito precio, quod inter nobis bona voluntatem convenit, pro servo uno iuris meis auem abere viso sum. nomine Martiro. subdiaconus ut exxead de $\mathrm{m}^{\circ} \mathrm{o}$, oui sunra Daniel, nresbiter, vel de meis beredibus, dominium et notestatem et deveniad in vos, qui sunra domnus Audax episcopus, vel ad vestris heredibus dominarionem et notestatem a presenti die et ora dominium et notestatem et de iamdicto Martino. servo meo, faciendi et iudicandi vos, oui subra domnus Audax, enisconus, vel vestris heredihus. iure nronrietario nomine lega'iter quitquid volueritis sine omni mª, qui supra Daniel nresbiter. vel eredum maorum contradictione: ita et libere ego, qui sunra Daniel, presbiter, vobis, qui supra domnus Audax enisconus, inso iamdicto Martino suhdiaconus, servo moo pro iam dicto precio a nresenti die vindedi, mancinavi et tradedi seu et investivi Der anc cartola vindicionis. $\mathrm{rum}$ omnem corauisto suo. totum ex integrum. Et snordeo me ego qui sunra, Daniel presbiter, vel meis heredibus, vobis, qui supra domnus Audax enisconus, vel ad vestris heredibus iam dicta vindicione ab omni omines defensare, et auod, si menime defendere potuerimus, aut vos quoaue temnore molestaverimus, dublis bonis condicionibus vobis restituamus re melioratas personas sicut in eodem temoore anut vos melioratas fuerint et pronter onorem sacerdotale mei quam pro ambliore firmitatem. set volo quod voluit auod a me semel factum vel conscrintum est inviolabiliter conservare promitto. Quam igitur cartola vindicionis Ledo notarius scrivere sic rogavi cum stinulacione subnixsa roboranda. Actum in Aste Civitate mense et indictione iamdicta feliciter.

Ego Daniel presbiter in anc cartola a me facta mea manu subscripsi" (Historiae Patriae Monumenta, t. I, col. 127). 
C. VERLINDEN, professeur ordinaire à l'Université de Gand, Directeur de l'Academia Belgica et de l'Institut Historique belge à Rome. Membre de l'Académie royale flamande des Sciences de Belgique, de l'Academia de la Historia de Madrid, de l'Instituto de Coimbra, de la Sociedad Peruviana de Historia à Lima, de la Comisión Panamericana de Historia à Mexico, de la Royal Historical Society de Londres, de la Medieval Academy des Etats Unis, de la Società Nazionale di Scienze, Lettere de Arti (Classe des Sciences morales) d'Italie. Docteur honoris causa de l'Université de Séville. Titulaire du Prix International Galileo Galilei. Auteur de 22 volumes et de très nombreux articles d'histoire médiévale et moderne ainsi que d'histoire de la colonisation. Ses livres les plus importants sont L'esclavage dans l'Europe médiévale ( 1 volume paru, un second en préparation) et Documents pour l'histoire des prix et des salaires en Flandre et en Brabant (XIVe-XIXe siècles) ( 5 volumes en collaboration). A fait des cours et des conférences dans 45 universités d'Europe et d'Amérique. 